\title{
A Lattice for the Muon Collider Demonstration Ring in the RHIC Tunnel *
}

\author{
D. Trbojevic, R.B. Palmer, E.D. Courant, J. Gallardo, S. Peggs, S. Tepikiañ E EIVED
Brookhaven National Laboratory, Upton, NY, 11973, USA \\ K. Y. Ng, Fermi National Laboratory

\section{Abstract}

The future $\mu^{+} \mu^{-}$Muon Collider should have a luminosity of the order of $10^{35} \mathrm{~cm}^{-2} \mathrm{~s}^{-1}$, an the energy of $2 \times 2 \mathrm{TeV}$. We present here a demonstration machine at a lower energy to test the feasibility of all components involved, which could be placed inside the existing Relativistic Heavy Ion Collider (RHIC) tunnel. The maximum energy of the muons in the RHIC tunnel depends on the maximum attainable field in the dipoles. The maximum energy in the existing RHIC rings for protons is $250 \mathrm{GeV}$, where the strength of the magnetic field in the dipoles is $3.5 \mathrm{~T}$. A design of the storage ring lattice for a $50 \mathrm{GeV}$ muon demonstration machine is also presented.

\section{INTRODUCTION}

The Muon Collider advantage with respect to an electron collider at high energies is that it could be a circular machine, because of negligible synchrotron radiation (for energies less than $10 \mathrm{TeV}$ ). This makes the number of collisions per bunch much larger than 1 in linear electron colliders, and together with a larger mass makes, in principle, a luminosity advantage of a factor of $10^{5}$ for the same size machine. Other components of the muon collider, its feasibility and physics opportunities are presented in reports [1],[2], [3], [4], [5], [6 etc. The muon collider storage ring will have $2 \mathrm{TeV}$ energy $\mu^{+}$and $\mu^{-}$bunches and will provide luminosity of $10^{35} \mathrm{~cm}^{-2} \mathrm{~s}^{-1}$. For this luminosity the rms bunch length has to be $3 \mathrm{~mm}$, which defines the $\beta^{*}=3 \mathrm{~mm}$ at IP. Short bunches require the collider to be isochronous [7]. In this report lattices for demonstration of the muon collider rings, with muon energies of $250 \mathrm{GeV}$ and $50 \mathrm{GeV}$, are presented. Parameters for the demo machine (Section 1), like transverse and longitudinal emittances, distances of the first quadrupoles to the crossing point, etc. are defined first. Next, possible choices for values of the magnet apertures, lengths, and strength will be presented. The momentum spread of the $\mu^{+}$and $\mu^{-}$ beams sets up a limit for the dispersion function excursions. An existing ring and elements of the Relativistic Heavy Ion Collider (RHIC) were used to create a first example (Section 3) of an isochronous storage ring. The RHIC collision parameters and magnet elements were used for the new lattice. Next, a demo muco collider storage ring lattice, with $0.5 \mathrm{TeV}$ center of mass energy, is set inside the RHIC tunnel (Section 4). Finally another demo machine with a 0.1 Tev center of mass energy is presented (Section 5).

\footnotetext{
- Wort pexformed uader the ampices of the US. Deparument of Exensy
}

\section{STORAGE RING PARAMETE}

Muons, $\mu^{+}$and $\mu^{-}$, will be created from the decay of pions $\pi^{+}$and $\pi^{+}$, made from higher energy protons on a high $Z$ target. Muons, $\mu^{+}$and $\mu^{-}$, will reach the collider storage ring after they have been accelerated and cooled in both longitudinal and transverse phase space.

\begin{tabular}{||c|c|c|c|c||}
\hline Table 1 \\
\hline E (TeV) & $\Delta p / p(\%)$ & $\epsilon(\pi \mathrm{m})$ & $\beta^{*}(\mathrm{~mm})$ & $L(\mathrm{~m})$ \\
\hline 4.0 & 0.12 & $5010^{-6}$ & 3 & 6.50 \\
0.4 & 0.12 & $8510^{-6}$ & 10 & 4.00 \\
0.2 & 0.12 & $10810^{-6}$ & 13 & 2.80 \\
0.1 & 0.12 & $13610^{-6}$ & 16 & 2.00 \\
\hline
\end{tabular}

A design of a demonstration muon collider storage ring would be at much lower muon energy than the proposed $4 \mathrm{TeV}$ (center of mass energy) machine. The muon sixdimensional emittance, defined as:

$$
\varepsilon_{6 D}=\varepsilon_{x}^{N o r m} \varepsilon_{y}^{N o r m} \Delta p / p \sigma_{z} \gamma \beta
$$

of a lower energy demo ring should not be different from the estimated $4 \mathrm{TeV}$ collider emittance (latest prediction $\left.\varepsilon_{6 D}=170 \cdot 10^{-12} \pi \mathrm{m}\right)$. A free space parameter $L D$, between the first high focusing quadrupole and the crossing collision point, is defined by the opening angle of the detector. The bunch length defines the $\beta^{*}$ at the crossing point. The momentum compaction $\alpha\left(\alpha=1 / \gamma_{t}^{2}\right)$ also depends on the bunch length. Parameters of interest for a lattice design of lower energy storage rings, are presented in Table 1. The bunch lengths in the $200 \mathrm{GeV}$ and $50 \mathrm{GeV}$ demo muon colliders, as presented in Table 1, are equal to $10 \mathrm{~cm}$ and $16 \mathrm{~cm}$, respectively. Short bunches require the momentum compaction to be adjusted. The maximum of the dispersion function in the lattice design is limited by the large beam momentum spread. Momentum offsets of the incoming muons are estimated to be large $(\Delta p / p=0.12 \%)$. If the beam size, resulting from the momentum offset, $\sigma_{p}$, is of the order of the transverse beam size, defined by the betatron function, $\sigma_{\beta}$, than:

$$
D_{\max } \leq \sigma /(\Delta p / p)
$$

The muons have a short life time. Products of the muon decay deposit energy in the magnets, requiring shielding of the superconducting magnets. The size of the aperture is reduced and has to be considered in the lattice design. The aperture of the magnets depends on the muon beam size. 
Table 2

\begin{tabular}{||c|c|c|c|c||}
\hline E TeV & Shield & Coil $(\mathrm{cm})$ & $P_{l}(\mathrm{~W} / \mathrm{m})$ & $L\left(\mathrm{~s}^{-1} \mathrm{~cm}^{-2}\right)$ \\
\hline 4.0 & $\mathrm{R}=6.0 \mathrm{~cm}$ & $\mathrm{D}=8.0$ & 1292 & $73210^{32}$ \\
0.4 & $\mathrm{R}=4.0 \mathrm{~cm}$ & $\mathrm{D}=6.0$ & 431 & $8.410^{32}$ \\
0.2 & $\mathrm{R}=3.5 \mathrm{~cm}$ & $\mathrm{D}=5.5$ & 431 & $2.610^{32}$ \\
0.1 & $\mathrm{R}=3.0 \mathrm{~cm}$ & $\mathrm{D}=5.0$ & 431 & $0.810^{32}$ \\
\hline
\end{tabular}

Parameters like: the shield radius, quadrupole coil diameter, power per length dissipation, and luminosities at four different center of mass muon energies, are presented in Table 2:

\section{ISOCHRONOUS RHIC LATTICE}

The RHIC collider lattice is three fold symmetrical. It has standard FODO cells in the arcs and six "zero" dispersion anti-symmetric interaction regions (IR) with triplet quadrupoles. Dipoles reach a magnetic field of $3.5 \mathrm{~T}$ with $30 \%$ quench margin in the arcs. An isochronous lattice is designed by using flexible momentum compaction (FMC) modules, as previously presented [8], and magnets of the same size as in the present RHIC machine. Figure 1 shows the betatron functions inside one of the IRs together with one FMC module.

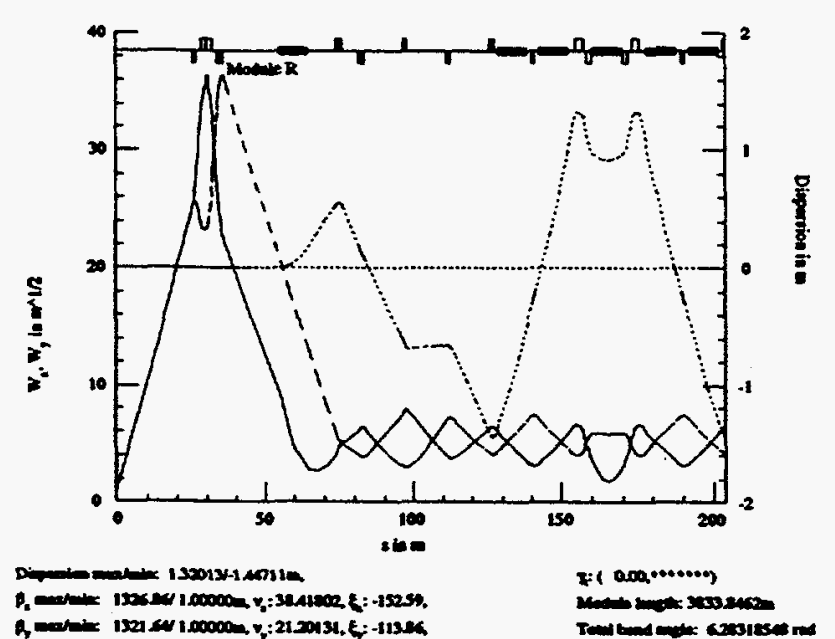

Figure 1: Isochronous RHIC lattice-IR with one FMC module.

\section{GEV ISOCARONOUS MUON DEMO COLLIDER LATTICE IN THE RHIC TUNNEL}

Previous RHIC upgrade parameters at the crossing points, of $\beta^{*}=1 \mathrm{~m}$ and $\beta_{\max }=1326 \mathrm{~m}$, are replaced by the $250 \mathrm{GeV}$ demo muon collider storage ring of $\beta^{*}=10$ $\mathrm{mm}$ and $\beta_{\text {mex }}=18525 \mathrm{~m}$. The distance between the crossing point and the first quad is estimated to be $L D \sim 3 \mathrm{~m}$. The RHIC triplet is replaced by quadrupole magnets optimized for the demo muon collider. Figure 2 shows the betatron functions inside one of the IRs together with one FMC module. The FMC module is the same as in the provious example. A dipole, located between the first and the second high focusing quadrupoles, reduces the background of the detector.

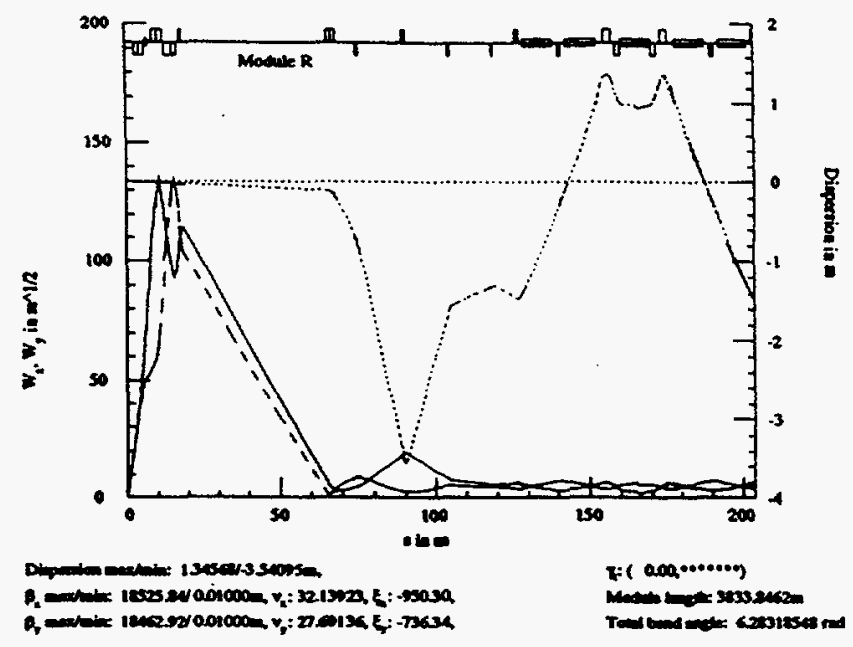

Figure 2: The IR and one FMC module of the $250 \mathrm{GeV}$ isochronous demo muon collider lattice in the RHIC tunnel.

\section{DEMO MUON COLLIDER 50 GEV STORAGE RING}

The rms transverse emittance of a $50 \mathrm{GeV}$ on $50 \mathrm{GeV}$ muon storage ring is estimated to be $\varepsilon=136 \cdot 10^{-6} \pi \mathrm{m}$ (see Table 1). The beam size is defined as:

$$
\sigma=\sqrt{\sigma_{\beta}^{2}+\sigma_{p}^{2}}
$$

while from the rms transverse emittance:

$$
\sigma_{\beta}=\sqrt{\frac{\varepsilon \beta_{\text {Twise }}}{\gamma \beta} .}
$$

The relativistic factor for a muon energy of $50 \mathrm{GeV}$ is $\gamma \beta=473.22$. The rms muon beam momentum width reaching the $50 \mathrm{GeV}$ demo collider is estimated to be $\Delta p / p=$ $0.12 \%$. If the maximum value of the dispersion function is $D_{\max }=2 \mathrm{~m}$, the beam size $\sigma_{p}=D_{\max } \Delta p / p=2.4 \mathrm{~mm}$. For $D_{\text {max }}=1 \mathrm{~m}$ the rms of beam size from the momentum width is a factor of two smaller $\left(\sigma_{p}=1.2 \mathrm{~mm}\right)$. If the maximum pole tip field at the quadrupoles is $B_{\text {mex }}=8 \mathrm{~T}$, then estimated gradients are calculated by using the radii equal to $4 \sigma$.

The demo muon collider storage ring consists of eight FMC modules, which make the arc part of the machine, and a single interaction region with one crossing point. The FMC module consists of four FODO cells with a $\pi$ low beta section in the middle. Longitudinal dimensions of the magnets inside the FODO cells define the maximum values of the betatron functions. There has to be enough clearance between the wall of the beam pipe, which is reduced due to the shield and the circulating beam. Construction of the FMC module starts by defining a $90^{\circ}$ FODO cell which conforms to the $\sigma_{\beta}$ beam size request. The maximum value of the dispersion function in this FODO cell is defined by the size of the bending angle. As reported carlier [9] the dispersion value in an FMC module is roughly half of the value within the $90^{\circ}$ FODO cell. The length of a dipole in 
the FMC module is $L_{d}=3.7 \mathrm{~m}$. The maximum values of the betatron functions in the FMC module are $\beta_{x}=17.1 \mathrm{~m}$ and $\beta_{y}=17.5 \mathrm{~m}$, and for the dispersion function $D_{x}=+1.38 \mathrm{~m}$ and $D_{x}=-1.23 \mathrm{~m}$, as presented in Figure 3. A proce-

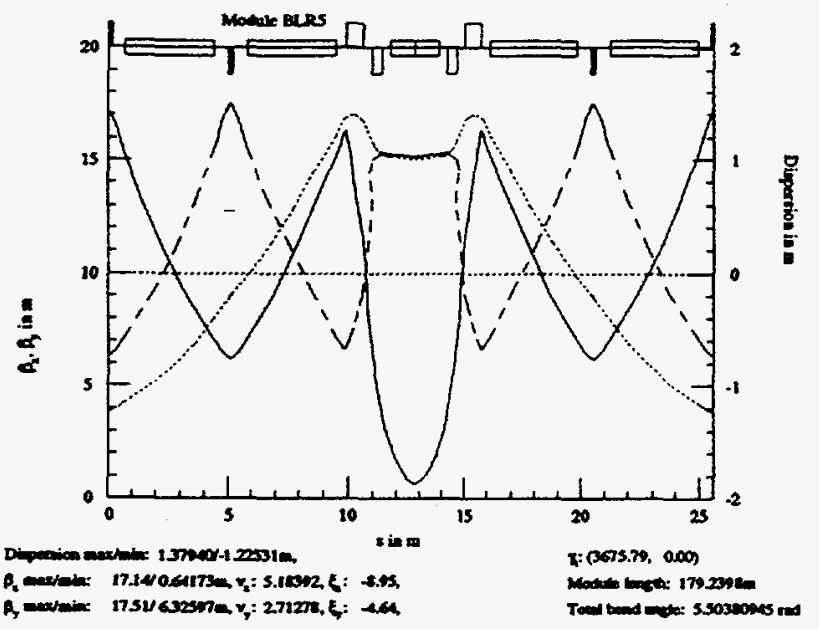

Figure 3: The Flexible Momentum Compaction Module of the $50 \mathrm{GeV}$ demonstration storage ring

dure for the interaction region triplet design was previousiy reported [10]. Table 3 shows the quadrupole gradients and lengths:

Table 3

\begin{tabular}{||c|c|c|c|c|}
\hline QUAD & $\mathbf{G}(\mathbf{l} / \mathrm{m})$ & Length(m) & $\hat{\beta}_{x}(\mathrm{~m})$ & $\beta_{y}(\mathrm{~m})$ \\
\hline Q1 & -115.0 & 0.686 & 597.0 & 331 \\
Q2 & 58.34 & 2.293 & 2261.3 & 1140.0 \\
Q3 & -58.34 & 1.646 & 1083.15 & 2261.0 \\
Q4 & 123.0 & 1.630 & 135.54 & 2.03 \\
\hline
\end{tabular}

It is important to note that for experimental background reduction a dipole was installed between the first and the second quadrupoles inside the IR region, and that the quadrupoles on the opposite sides of the crossing point are antisymmetric to balance the chromaticities. The betatron functions of the interaction region together with FMC modules are presented in Figure 4. The circumference of the machine is $263.4 \mathrm{~m}$ with an average radius of $42 \mathrm{~m}$. There are eight FMC modules each $25.6 \mathrm{~m}$ long, and one straight section-IR $58.5 \mathrm{~m}$ long. The chromaticity of the whole lattice is corrected by sextupoles within the FMC modules in the arcs. The second order tune shift induced by the sextupole is small (all $\alpha_{x x}, \alpha_{x y}, \alpha_{y y} \leq 10^{4} \mathrm{~m}^{-1}$ ).

\section{SUMMARY}

A progress report on the muon collider demonstration machines was presented. First, two examples showed that an isochronous storage ring could be placed inside an existing tunnel (RHIC) with magnets already built. The center of mass energy for $\mu^{+}$and $\mu^{-}$beams in these two rings is 0.5 TeV. If this energy is selected for a demonstration machine, the circumference of the new ring could be much smaller than in the examples presented. The two examples of 250

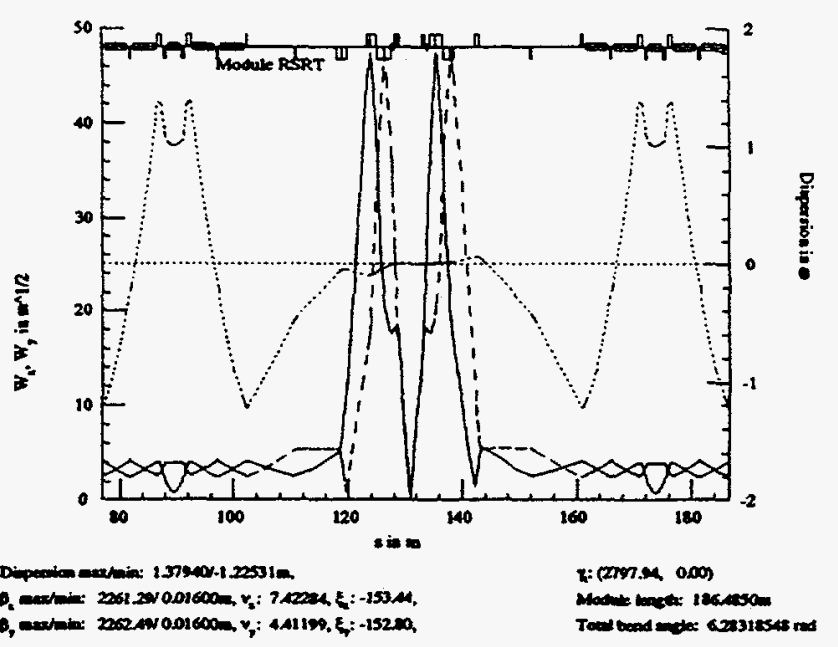

Figure 4: the IR with two FMC modules of the $50 \mathrm{GeV}$ isochronous demo muon collider lattice.

$\mathrm{GeV}$ storage rings had a circumference $3833 \mathrm{~m}$ because this is the RHIC circumference. The size of the $0.1 \mathrm{TeV}$ muon collider demonstration storage ring is very small (the circumference is only $263 \mathrm{~m}$ ). More tracking and detector background studies will follow.

\section{REFERENCES}

[1] R.B. Palmer, "Progress on $\mu^{+} \mu^{-}$Colliders", these proceedings.

[2] D. Trbojevic, J.M. Brennan, E.D. Courant, T. Roser, S. Peggs, J. Norem, C. Johnstone, K.Y. Ng, M. Popovic, "Proton Driver Lattice for the Muon Collider Source with Adjustable Momentum Compaction", these proceedings.

[3] J. Norem, C. Ankenbrandt, J. Griffin, C. Johnstone, F. Mills, A. Moretti, R. Noble, M Popovic, Z Qian, M. Brennan, T. Roser, D. Trbojevic, J. Wei, "The Proton Driver for the $\mu^{+} \mu^{-}$Collider", these proceedings.

[4] D. Neuffer and A.Van Ginneken, " $\mu$-Ionization Cooling Simulation Results", these proceedings.

[5] R.C. Fernow, J.C. Gallardo, R.B. Palmer, "Ionization Cooling using a FOFO lattice", these proceedings.

[6] D. Summers, D. Neuffer, Q.S. Shu, and E. Willen, "Acceleration for the $\mu^{-} \mu^{+}$. Collider", these proceedings.

[7] D. Trbojevic, K.Y. Ng, E.D. Courant, S.Y. Lee, C. Johnstone, J. Gallardo, R.B. Palmer, and S. Tepikian, "Design of the Muon Collider Isochronous Storage Ring Lattice", Micro-Bunches Workshop, AIP Press, Conference Proceedings, (1996), pp. 269-278.

[8] S. Y. Lee, K.Y. Ng, D. Trbojevic, "Minimizing dispersion in fiexible-momentum compaction lattices", Physical Review E, Vol. 48, Number 4, October 1993, pp. 3040-3048.

[9] D. Trbojevic, K.Y.Ng, and S.Y. Lee, "Is the Momentum Space Optimally Used with the FODO Lattices". Stability of Particles in Storage Rings, Particles and Fields, Series 54, AIP Conference Proceedings No.292, pp. 260-263.

[10] R.B. Palmer et al., "Muon Colliders", in Beam Dynamics and Technology Issues for $\mu^{+} \mu^{-}$Collider, AIP Conference Proceedings 372, American Insitute of Physics, 1996, p.21. 


\section{DISCLAIMER}

This report was prepared as an account of work sponsored by an agency of the United States Government. Neither the United States Government nor any agency thereof, nor any of their empleyces, makes any warranty, express or implied, or assumes any legal liability or responsibility for the accuracy, completeness, or usefulness of any information, apparatus, product, or process disclosed, or represents that its use would not infringe privately owned rights. Reference herein to any specific commercial product, process, or service by trade name, trademark, manufacturer, or otherwise does not necessarily constitute or imply its endorsement, recommendation, or favoring by the United States Government or any agency thereof. The views and opinions of authors expressed herein do not necessarily state or reflect those of the United States Government or any agency thereof. 


\section{DISCLAMIER}

Portions of this document may be illegible in electronic image products. Images are produced from the best available original document. 